\title{
Retention of an incompletely learned avoidance response: Some problems with replication
}

\author{
D. CHRIS ANDERSON, L. JOHNSON ${ }^{1}$, G. SCHWENDIMAN AND G. DUNFORD
}

BRIGHAM YOUNG UNIVERSITY

Eight experiments, each designed to determine the cause of failure to replicate the "Kamin-effect," were performed. Replication was finally obtained for Experiments VII and VIII in which original avoidance training was carried to only one successful avoidance. Inder this criterion there was little or no overlap in the combined performances of groups retrained at .08 and $24 \mathrm{hr}$. intersession intervals with those retrained at 1 and $4 \mathrm{hr}$. periods.

Kamin (1957; 1963) and others (Brush, Myer, \& Palmer, 1963; Denny \& Ditchman, 1962; Pinckney, 1966; Tarpy, 1966) have reported that retention of an incompletely learned avoidance response is a U-shaped function of intersession interval, being best at $5 \mathrm{~min}$. and $24 \mathrm{hr}$. following original training and significantly depressed for the intermediate times of 1 and $4 \mathrm{hr}$. Our original intention was to evaluate the motivationalphysiological properties of the CS at the various prescribed intersession intervals. To accomplish this, it was first necessary to establish the "Kamin-effect" in our laboratory. The difficulties encountered and the resolution of them in demonstrating this phenomenon are reported below.

\section{Method}

A total of eight studies are summarized. The number of Ss per group are identified in Fig. 1 and have been drawn in over each respective curve (each curve represents a separate study). The Ss were naive male albino rats. The apparatus was a 5 in. wide $\times 15$ in. high $x$ 24 in. long (inside) shuttlebox. Located at either end and 12 in. above the floor was a $7-1 / 2$ w lamp. Located immediately beneath the center of the apparatus was a $6 \mathrm{v}$ buzzer. The CS was an intermittent buzzer-light complex (.21 sec. on; .12 sec. off). Shock source consisted of the output of a variable autotransformer through a $20 \mathrm{~K}$ series-connected resistor. Shock was scrambled and switching and timing circuitry were programmed by a series of photo-relay devices and silenced electronic timers.

The general procedure for all eight experiments paralleled that described by Kamin (1963).

Experiment I following the initial adaptation period to the shuttle apparatus, Ss were trained to a criterion of three successful avoidances. Ss were then removed from the apparatus, matched on the basis of total trials to criterion, and then assigned to one of four groups corresponding to respective intersession intervals $(0.08,1,4,24 \mathrm{hr}$.). At the respective intervals Ss were returned to the shuttle apparatus and administered 40 additional training trials. Shock level was $75 \mathrm{v}$. In addition, a hurdle was located at the center of the apparatus for both the original and subsequent training sessions.

Experiment II was an exact replication of Experiment I except that US intensity was reduced to $45 \mathrm{v}$.

Experiment III was an exact replication of Experiment II except that the hurdle was removed and $3 / 4$ in. inserts were substituted to demarcate the compartments.

Experiment IV was an exact replication of Experiment III except that the grids were rewired so that each side of the apparatus was electronically isolated from

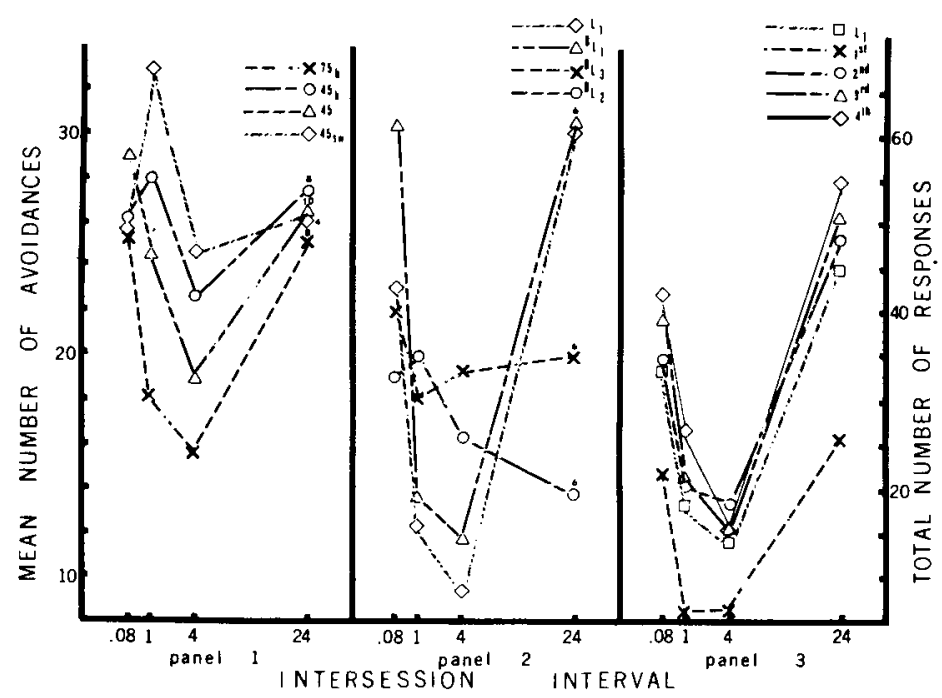

Psychon. Sci., 1966, Vol. 6 (1)
Fig. 1. Panels 1 and 2 represent mean number of avoidances in a shuttlebox for different groups $.08,1,4$, or 24 hr. following original learning in the same situation. Group $75_{h}$ was trained and tested under $75 \mathrm{v}$ with a hurdle; Group $45_{h}$ under 45 v; Group 45 without a hurdle; Group $45_{\mathrm{sw}}$ with each side independently wired; Group $B_{\text {L3 }}$ with only a blinking light $\mathrm{CS}$ and to three avoidances during original leaming: Group $B_{L_{2}}$ to two avoidances; Group $B_{\text {LI }}$ to one avoidance; and Group $L_{1}$ to one avoidance with a non-intermittent light CS. Panel 3 represents total number of responses for the 1st, 2nd, 3rd, and 4th block of 10 trials for each group originally trained with a non-intermittent light $\mathrm{CS}$. 
the other and could be separately switched.

Experiment $V$ was identical to Experiment IV except that the buzzer component of the CS was eliminated.

Experiment VI duplicated Experiment V except that the original training criterion was reduced from three to two successful avoidance responses.

Experiment VII replicated Experiment VI except that the original criterion was reduced from two to one correct avoidance responses.

Experiment VIII was identical to Experiment VII with the exception that the intermittent light CS was made continuous.

\section{Results}

Figure 1 graphically summarizes the results of all eight experiments. The first four experiments are represented in panel 1 . The most general statement that can be made of these four studies is that the effects of intersession interval did not produce a significant $F$ for any of them. Indeed, while the graphic display for Experiment I most closely approached a U-shaped function, the $F$ for intersession interval effects was barely larger than $1(F=1.18, d f=3 / 28, p>.05)$.

A graphic comparison of Experiments $V$ through VIII is presented in panel 2 of Fig. 1. Several important conclusions are summarized in this panel and are supported by appropriate analyses. Intersession interval was not a significant source of variance for either Experiment V or VI. However, intersession interval emerged as a highly significant effect for Experiments VII $(F=19.01, \mathrm{df}=3 / 20, \mathrm{p}<.001)$ and VIII $(\mathrm{F}=8.49$, $\mathrm{df}=3 / 20, \mathrm{p}<.001$ ). It should be noted that, as with the prior six studies, the four groups of Experiments VII and VIII, respectively, did not differ in terms of number of trials to original training criterion, (Fs $<1)$. Interestingly, the mean number of trials to the first successful avoidance, collapsing over the eight groups of Experiments VII and VII, was 4.4. This value is considerably smaller than the number of trials to first avoidance reported by other investigators (cf. Brush, Myer, \& Palmer, 1963).

Panel 3 represents the total number of responses for each of four blocks of 10 trials for the respective groups of Experiment VIII. As can be seen, the graphic display for each block of trials roughly parallels the form of the original U-shaped function indicating that the "Kamin-effect" is not confined to any particular segment of the retraining session.

A final comment to be made is that for both Experiments VII and VIII there was little or no overlap between the combined performances of the $5 \mathrm{~min}$. and $24 \mathrm{hr}$. groups and those of the two intermediate groups, a finding apparently unique to our procedure.

\section{Discussion}

Our data (Experiments VII and VIII) clearly replicate the "Kamin-effect" and show that it is obtainable with a very weak criterion of original learning and under a wide variety of conditions. The data do not show, however, why we were unable to obtain the phenomenon under the more standard conditions described by Kamin and others. Failure to replicate the findings of others probably can not be ascribed to apparatus differences since (1) the dimensions of our shuttlebox were similar to the specifications described as optimal by Denny \& Thomas (1963), and (2) the general nature of our CS, shock circuitry, and timing and measuring devices roughly paralleled those employed by Kamin and others. Further, it also seems unlikely that our results can be explained in terms of strain differences since (1) there have been numerous reports of successful replications by investigators who employed widely disparate strains and species raised in widely disparate geographic locations and (2) we have employed strains of rats used successfully by others.

We believe our data call for a "response discrimination" hypothesis which suggests that the more "discriminable" a response, the less likely the effect will be obtained. It may be that certain subtle features of our apparatus contributed to more rapid learning, and, hence, that three successful avoidances in our situation was analogous to more massive training in the situations employed by other investigators.

\section{References}

Brush, F. R., Myer, J. S., \& Palmer, M. E. Effects of kind of prior training and intersession interval upon subsequent avoidance learning. J. comp. physiol. Psychol., 1963, 56, 539-545.

Denny, M. R., \& Ditchman, R. E. The locus of maximal "Kamineffect" in rats. J. comp. physiol. Psychol., 1962, 55, 1069-1070.

Denny, M. R., \& Thomas, J. O. Avoidance learning and relearning as a function of shuttlebox dimensions. Science, 1960, 132, 3247.

Kamin, L. S. The retention of an incompletely learned avoidance response. J. comp. physiol. Psychol, 1957, 50, 457-460.

Kamin, L. S. Retention of an incompletely learned avoidance response: some further analyses. J. comp. physiol. Psychol., $1963,56,713-718$.

Pinckney, G. A. The Kamin effect in fish. Psychon. Sci., 1966, 4, 387-388.

Tarpy, R. M. Incubation of anxiety as measured by response suppression. Psychon. Sci., 1966, 4, 189-190.

Note

1. This author was an NSF-URP undergraduate fellow during participation in these experiments. Now at Tulane University. 